\author{
Abramova V.A., Kali A., Abdolla N., Yurikova O.Yu., Perfilyeva Yu.V., \\ Ostapchuk Ye.O., Tleulieva R.T., Madenova S.K., Belyaev N.N.
M.A.Aitkhozhin Institute of Molecular Biology and Biochemistry, Almaty
*E-mail: mglory91@mail.ru

\title{
Influence of tumor cells on natural killer cell phenotype and cytotoxicity
}

\begin{abstract}
Natural Killer (NK) cells are known to lyse tumor cells lacking MHC-I and expressing ligands for activating receptors, thereby participating in cancer immune surveillance. However, their function and phenotype change significantly in cancer patients. The precise molecular mechanism(s) involved in this phenomenon remain unknown. The aim of the present study was to investigate changes of both receptor phenotype and cytotoxic activity of NK cells cocultured with K562 and HepG2 cells in dual-chamber Transwell ${ }^{\circledR}$ plates. A decrease of NK cell cytotoxicity against K562 cells was demonstrated. This effect was not a result of changes in perforin, granzyme or CD107a expression by NK cells or by production of cytokines (IFN $\gamma, \mathrm{TNF} \alpha$, IL-10, and TGF $\beta$ ). No changes in the expression of a majority of NK cell receptors (CD16, CD69, 2B4, NKG2A, NKG2D, NKp30, NKp44, NKp46, and DNAM1) were observed. Only an increase in the percentage of NK cells bearing inhibitory receptor TIGIT was found as a result of co-culture with tumor cells. Possible molecular mechanisms of altered NK cell cytotoxicity involving TIGIT are discussed.
\end{abstract}

Key words: NK cells, cytotoxicity, cancer immune surveillance.

\section{Introduction}

Natural Killer (NK) cells play a key role in immune surveillance in cancer. They dispatch susceptible cells by various mechanisms, including antibody-dependent cellular cytotoxicity, receptormediated induction of apoptosis, and triggered release of cytotoxic granules containing perforin and granzyme [1]. Initiation of killer NK cells begins with recognition of specific ligands expressed on target cells by a panel of receptors functionally categorized as activating or inhibitory. The NK cell response represents a net result of the interplay of signals from these activating and inhibitory receptors [2].

NK cell function and phenotype are severely altered in various types of malignant tumors, such as colorectal, breast, lung, hepatic, and others [3-6]. Various soluble factors secreted by tumor cells and their immunosuppressive microenvironment, including Treg cells, myeloid-derived suppressor cells, tolerogenic dendritic cells, and others, contribute to these changes [3]. However, NK cell function and phenotype change significantly in cancer patients, and the molecular mechanism(s) involved remain unknown.

The aim of the present study was to study changes of both receptor phenotype and cytotoxic activity of human NK cells co-cultured with K562 and HepG2 cells in dual-chamber Transwell ${ }^{\circledR}$ plates. Results revealed a decrease of NK cell cytotoxicity under the influence of soluble tumor factors, accompanied by an increase in the percentage of NK cells expressing inhibitory receptor TIGIT.

\section{Materials and methods}

\section{Blood samples and cell lines}

This study was approved by relevant institutional review boards and all volunteers gave written informed consent according to the Helsinki Declaration. Peripheral blood samples were obtained from healthy volunteers. Human erythroleukemia K562 and hepatocarcinoma HepG2 cell lines were purchased from ATCC (USA). Cells were cultured in complete culture medium consisting of RPMI1640 supplemented with $10 \%$ fetal calf serum, 2 $\mathrm{mM}$ glutamine, $100 \mathrm{U} / \mathrm{ml}$ penicillin, and $100 \mathrm{mg} / \mathrm{ml}$ 
streptomycin (Sigma-Aldrich, USA) at $37^{\circ} \mathrm{C}$ and $5 \% \mathrm{CO}_{2}$. Before use, adherent HepG2 cells were detached from Petri dishes with a $0.05 \%$ trypsin solution and washed in RPMI-1640 medium.

\section{Antibodies}

The following labeled anti-human monoclonal antibodies (mAbs) were used for flow cytometry: anti-CD3-FITC, anti-CD56-PE, anti-CD56-PerCP, anti-CD107a-PerCP-Cy5.5, and anti-CD16-PE (Biolegend, USA); anti-NKp44-APC, anti-TIGITbiotin, anti-DNAM-1-APC, anti-NKp46-APC, antiCD69-FITC, anti-Perforin-APC, anti-NKp30-APC, anti-2B4-FITC, and anti-NKG2D-APC (Miltenyi Biotec, Germany); anti-NKG2D-PE and antiGranzyme-PE (BD Biosciences, USA); antiNKG2A-PerCP (R\&D Systems, USA). Streptavidin-PE (R\&D Systems, USA) was used for detection of cells linked to anti-TIGIT-biotin.

\section{Cell separation}

Peripheral blood mononuclear cells were isolated from venous peripheral blood by Hystopaque1077 (Sigma-Aldrich) density gradient centrifugation. NK cells were purified by immunomagnetic separation using an NK Isolation Kit and VarioMACS cell separator (Miltenyi Biotech) according to the manufacturer's instruction. The purity of $\mathrm{CD}^{-}{ }^{-} \mathrm{CD} 56^{+}$cell populations exceeded $90 \%$ as estimated by flow cytometry.

\section{Design of experiment in Transwell plates}

NK cells $\left(5 \times 10^{5}\right)$ were placed in the bottom chamber of dual-chamber Transwell ${ }^{\mathbb{R}}$ plates (Sigma-Aldrich) in $1750 \mu \mathrm{l}$ of complete culture media. Upper wells were filled with $250 \mu \mathrm{l}$ of culture media containing $5 \times 10^{5}$ HepG 2 or K562 cells. Only NK cells were incubated in control wells. Coculture lasted for $48 \mathrm{~h}$ at $37{ }^{\circ} \mathrm{C}$ and $5 \% \mathrm{CO}_{2}$, then NK cells were collected, labeled with mAbs, and subjected to flow cytometry. The following receptors were analyzed on gated $\mathrm{CD} 3{ }^{-} \mathrm{CD} 56^{+}$cells: CD16, NKG2A, NKG2D, NKp30, NKp44, NKp46, CD69, DNAM1, TIGIT, and 2B4.

\section{Flow cytometry}

For surface and intracellular staining, cells were first incubated with mAbs specific for surface markers according to the manufacturer's protocols, then fixed and permeabilized with Fixation/Permeabilization solution (BD Pharmingen, USA), mixed well, and incubated for $20 \mathrm{~min}$ in the dark at room temperature. Cells were then washed with Perm/Wash Buffer (BD Biosciences) and stained with $\mathrm{mAbs}$ specific for intracellular molecules. Afterwards, cells were washed with phosphate-buffered saline, resuspended in flow solution, and immediately analyzed by flow cytometry with FACSCalibur (BD Biosciences) and CellQuest software (BD Biosciences).

NK cell cytotoxicity assay

A 3-(4,5-dimethylthiazol-2-il)-2,5-diphenyltetrazolium bromide (MTT) assay was used to assess NK cell cytotoxicity. After incubation with K562 cells, HepG2 cells, or alone in Transwell ${ }^{\circledR}$ plates, NK cells were collected, counted, and a part of them were subjected to cytotoxicity assay. K562 cells were used as a target. Effector and target cells in a 2:1 ratio were co-cultured in round bottom 96well plates (Sigma-Aldrich) in $200 \mu$ of complete culture medium for $72 \mathrm{~h}$. Both NK and K562 cells alone were used as controls. Until the last $4 \mathrm{~h}$ of cultivation, $50 \mu \mathrm{l}$ of MTT stock solution (500 $\mu \mathrm{g} / \mathrm{ml}$; Sigma-Aldrich) was added. After cultivation, supernatants were removed and formazan crystals were dissolved in $150 \mu \mathrm{l}$ DMSO (SigmaAldrich) and absorbance determined at an optical density (OD) of 492/630 nm. The index of cytotoxicity (IC) was calculated as follows:

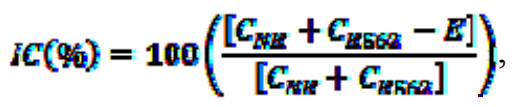

where $\mathrm{C}_{\mathrm{NK}}$ and $\mathrm{C}_{\mathrm{K} 562}$ are the OD of control wells and $\mathrm{E}$ is the OD of the experimental well.

NK cell cytotoxicity against K562 cells was also assessed by surface staining for CD107a and intracellular staining for perforin and granzyme B. For this, NK cells were co-cultured together with K562 cells as described above and then stained with anti-CD107a antibodies and incubated for $1 \mathrm{~h}$, after which Brefeldin A (Biolegend, USA) was added according to manufacturer's instruction. After 3 hours, cells were collected, washed with phosphate-buffered saline, and subjected to intracellular staining for perforin and granzyme B according to manufacturer's instruction.

\section{Cytokine secretion}

Supernatants from co-cultures in Transwell ${ }^{\circledR}$ plates were collected and frozen at $-20{ }^{\circ} \mathrm{C}$. Sandwich ELISA was performed using human IL-10, IFN $\gamma$ (BD Biosciences), and TGF $\beta 1$ (R\&D Systems) ELISA sets according the manufacturer's instructions.

\section{Statistical analysis}

Data are represented as mean values \pm quadratic error. The Student's t-test was used to analyze data. P-values of 0.05 were chosen as the limit of statistical significance. 


\section{Results and their discussion}

Analysis of NK cell cytotoxicity after co-culture with tumor cells

The cytotoxicity of NK cells against K562 cells is considered a gold standard for assessment of NK cell activity. In our study, the IC of NK cells after co-culture with HepG2 and K562 cells approached $52.2 \pm 9.3 \%$ and $53.4 \pm 10.3 \%$, respectively, contrary to $65.5 \pm 10.8 \%$ in the absence of co-cultured tumor cell lines. Since donor NK cells naturally differ by their primary cytolytic activity, we normalized IC values to controls (NK cells incubated alone in Transwell $^{\circledR}$ plates), which were considered as $100 \%$. Thereafter, NK cell cytotoxicity after incubation with both tumor cell lines reached 20\% (Fig. 1).

Perforin and granzyme, along with death receptor family ligands, are executioners of NK cell mediated cytotoxicity. CD107a is a marker of degranulation highly correlated with emptying or NK cell cytotoxic granules [7]. Unexpectedly, we did not find statistically significant differences in CD107a, perforin, or granzyme expression in NK cells cocultured with tumor cell lines (Table 1).

Phenotyping of NK cells

Transwell ${ }^{\mathbb{R}}$ plates allow free exchange of soluble factors and secreted exosomes between cocultured cells. We examined alterations of NK cell activating (DNAM-1, NKG2D, NKp30, NKp44, NKp46) and inhibitory receptors (TIGIT, NKG2A), as well as CD16 (FcR $\square \mathrm{III}$ ), involved in antibodydependent cellular cytotoxicity. However, changes in the expression of a majority of receptors/markers did not reach statistical significance (Table 2).

Taking into account the complex interplay between TIGIT and its activating counterpart, DNAM-1 [8], and their role in tumor surveillance, we analyzed independent and co-expression of DNAM-1 and TIGIT. A significant increase of NK cells expressing TIGIT alone after co-culture with both HepG2 and K562 cells was observed (Table 3 ). Levels of DNAM- ${ }^{+}$TIGIT $^{-}$and DNAM$1^{+}$TIGIT $^{+}$NK cells did not change significantly (Table 3, Fig. 2).

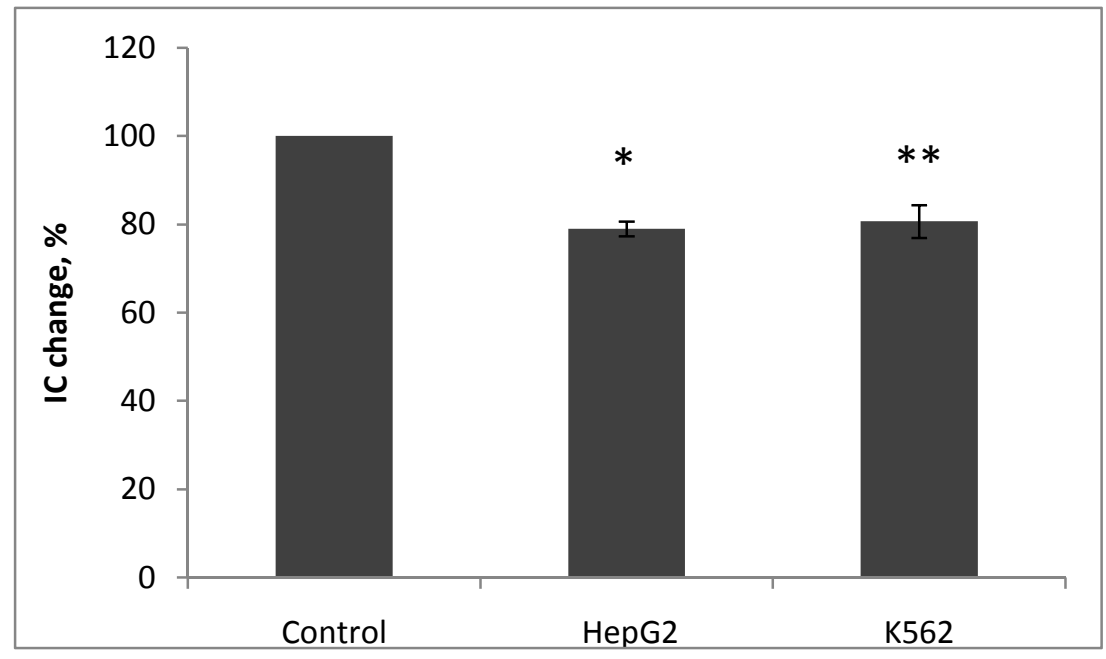

Figure 1 - Cytotoxicity of NK cells against K562 target after co-culture with HepG2 or K562 tumor cell line, assessed by MTT test. Data are normalized as percent of cytotoxicity index (IC) of NK cells, cultured in Transwell ${ }^{\circledR}$ plates alone, and are represented as $M \pm m, n=3$. $* \mathrm{P}=0.007, * * \mathrm{P}=0.038$

Table 1 - Percent of NK cells expressing molecules, associated with cytotoxicity

\begin{tabular}{|c|c|c|c|c|}
\hline Items & Variants of test & Granzyme & Perforin & CD107a \\
\hline 1 & NK alone & $85.9 \pm 2.7$ & $93.4 \pm 1.0$ & $99.2 \pm 0.4$ \\
\hline 2 & NK + HepG2 & $80.0 \pm 7.2$ & $89.2 \pm 2.8$ & $94.4 \pm 5.3$ \\
\hline 3 & $\mathrm{NK}+\mathrm{K} 562$ & $80.5 \pm 3.3$ & $89.6 \pm 1.8$ & $96.1 \pm 3.0$ \\
\hline 4 & $\mathrm{n}$ & 3 & 4 & 5 \\
\hline 5 & $\mathrm{P}_{1-2}$ & \multicolumn{3}{|c|}{$\mathrm{P}>0.05$} \\
\hline 6 & $\mathrm{P}_{1-3}$ & $\mathrm{P}>0.05$ & \\
\hline
\end{tabular}


Table 2 - Percent of NK cells expressing particular receptors

\begin{tabular}{|c|c|c|c|c|c|c|c|c|c|}
\hline Items & $\begin{array}{c}\text { Variants } \\
\text { of test }\end{array}$ & CD16 & CD69 & 2B4 & NKG2A & NKG2D & NKp30 & NKp44 & NKp46 \\
\hline 1 & NK alone & $69.9 \pm 11.5$ & $2.5 \pm 0.4$ & $86.5 \pm 21.7$ & $4.1 \pm 2.3$ & $76.3 \pm 5.6$ & $5.7 \pm 2.4$ & $1.8 \pm 0.8$ & $89.5 \pm 3.2$ \\
\hline 2 & NK+HepG2 & $79.8 \pm 1.3$ & $5.3 \pm 2.4$ & $81.2 \pm 10.2$ & $3.5 \pm 1.8$ & $76.6 \pm 8.7$ & $7.4 \pm 3.4$ & $2.9 \pm 1.3$ & $91.0 \pm 3.6$ \\
\hline 3 & NK+K562 & $73.4 \pm 6.0$ & $8.5 \pm 5.4$ & $82.8 \pm 10.5$ & $7.1 \pm 3.0$ & $69.9 \pm 11.6$ & $3.5 \pm 0.9$ & $2.7 \pm 0.6$ & $89.1 \pm 4.4$ \\
\hline 4 & $\mathrm{n}$ & 5 & 7 & 7 & 5 & 6 & 7 & 6 & 5 \\
\hline 5 & $\mathrm{P}_{1-2}$ & \multicolumn{7}{|c|}{$\mathrm{P}>0.05$} \\
\hline 6 & $\mathrm{P}_{1-3}$ & \multicolumn{7}{|c|}{$\mathrm{P}>0.05$} \\
\hline
\end{tabular}

Table 3 - Percent of NK cells, expressing DNAM-1, TIGIT or their combinations, by NK cells

\begin{tabular}{|c|c|c|c|c|}
\hline Items & $\begin{array}{c}\text { Variants } \\
\text { of test }\end{array}$ & DNAM-1 & TIGIT & DNAM- $^{+}$TIGIT $^{+}$ \\
\hline 1 & NK alone & $94.8 \pm 2.0$ & $4.7 \pm 1.4$ & $6.1 \pm 2.4$ \\
\hline 2 & NK+HepG2 & $94.2 \pm 1.2$ & $10.4 \pm 2.5$ & $16.9 \pm 5.1$ \\
\hline 3 & NK+K562 & $95.6 \pm 1.1$ & $12.0 \pm 2.5$ & $18.8 \pm 6.7$ \\
\hline 4 & $\mathrm{n}$ & 3 & 5 & 3 \\
\hline 5 & $\mathrm{P}_{1-2}$ & 0.811 & 0.042 & 0.204 \\
\hline 6 & $\mathrm{P}_{1-3}$ & 0.767 & 0.015 & 0.273 \\
\hline
\end{tabular}

\section{Analysis of cytokine production}

Cytokines are known to modulate NK cell activity. We analyzed participation of proinflammatory (IFN $\gamma, \mathrm{TNF} \alpha$ ) and anti-inflammatory (IL-10, TGF $\beta$ ) cytokines in modulation of NK cytotoxicity by determining their concentrations in no-culture supernatants. No changes in the concentration of any of these cytokines were found in the culture media after NK cell co-culture with tumor cells (Table 4).

The functional incapacitation of NK cells infiltrating tumors is well established [5]. Much re- search has been performed to analyze NK cell peculiarities in the presence of tumor cells in vitro and in cancer patients, but precise molecular mechanism(s) of this phenomenon remain unknown. Theoretically, two influences could alter NK cell cytolytic activity: cell-to-cell contact and/or the impact of soluble factors. In this study, we analyzed the influence of possible soluble factors secreted by two different tumor cell lines during co-culture with NK cells in dual-chamber Transwells ${ }^{\circledR}$ on NK cytolytic activity, as well as NK cell receptor/marker expression and cytokine production.

Table 4 - Cytokine levels in conditioned media, $\mathrm{pg} / \mathrm{ml}, \mathrm{M} \pm \mathrm{m}$.

\begin{tabular}{|c|c|c|c|c|}
\hline Items & $\begin{array}{c}\text { Variants } \\
\text { of test }\end{array}$ & IFN $\gamma$ & TNF $\alpha$ & IL-10 \\
\hline 1 & NK alone & $1579 \pm 43$ & $170 \pm 3$ & $257 \pm 8$ \\
\hline 2 & NK+HepG2 & $1630 \pm 11$ & $170 \pm 2$ & $266 \pm 4$ \\
\hline 3 & $\mathrm{NK}+\mathrm{K} 562$ & $1707 \pm 40$ & $182 \pm 6$ & $268 \pm 14$ \\
\hline 4 & $\mathrm{n}$ & 3 & 3 & 3 \\
\hline 5 & $\mathrm{P}_{1-2}$ & \multicolumn{3}{|c|}{} \\
\hline 6 & $\mathrm{P}_{1-3}$ & \multicolumn{2}{|c|}{} \\
\hline
\end{tabular}




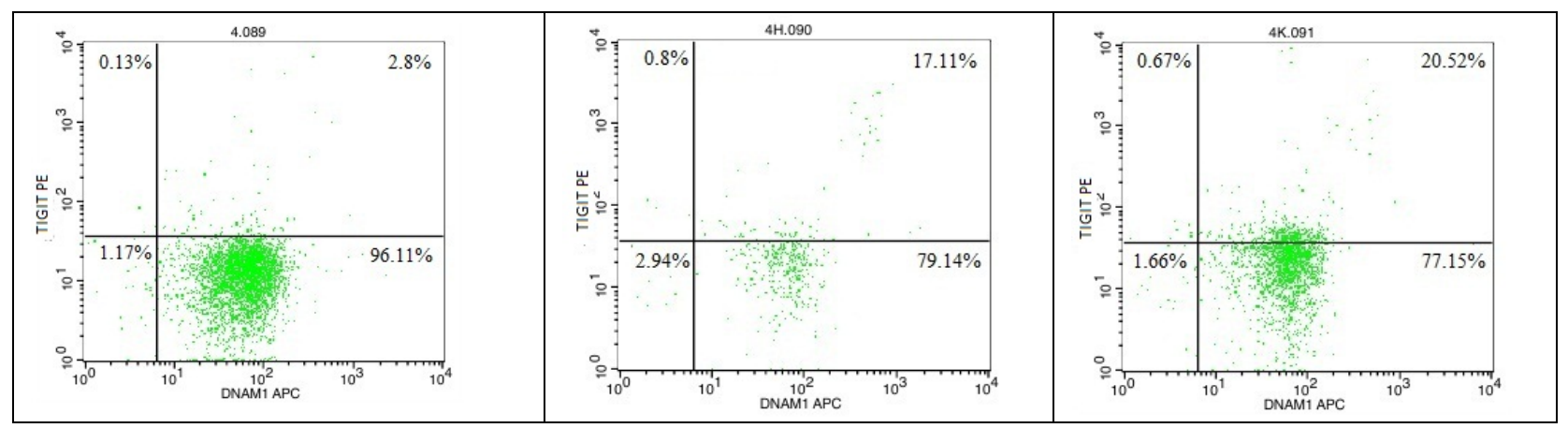

A

B

$\mathrm{C}$

Figure 2 - Representative data of flow cytometry showing co-expression of DNAM-1 and TIGIT on NK cells after Transwell ${ }^{\circledR}$ co-culture experiments. A - NK cells cultured alone; B - NK cells after co-culture together with HepG2 cells; NK cells after co-culture together with K562 cells

A decrease in NK cell cytotoxicity to K562 cells after co-culture with either HepG2 or K562 cells probably involved certain soluble tumor factors. We showed that the decrease of NK cell activity was not associated with an alteration in perforin and granzyme expression, two proteins known to mediate the cytolytic impact of NK cells. Moreover, we did not observe changes in CD107a presentation on the NK cell surface. These results indicate the fundamental readiness of NK cells to cytolytic activity.

Taken together, decreased NK cell function possibly results from deterioration of target ligand recognition by NK cell receptors due to inhibition of receptor expression. Our analysis of a panel of NK cell receptors (CD16, CD69, 2B4, NKG2D, NKp30, NKp44, NKp46) did not elucidate any changes in their expression after co-culture with tumor cell lines. In addition, there was no change in the expression of inhibitory receptor NKG2A. However, we did detect an increase in the expression of another inhibitory receptor, TIGIT, on NK cells after co-culture with HepG2 and K562 cells. This finding is of particular interest because the NK cell response is determined by the net outcome of signals from activating and inhibitory receptors [2]. Many NK cell activating or inhibitory receptors share a common ligand $[8,9]$. Distinctive examples are DNAM-1, TIGIT, and CD96, all critical regulators of NK cell function which share CD155 and CD112 ligands (members of nectin and nectin-like protein families) [10]. CD155 is a dominant ligand over CD112 for DNAM-1 and TIGIT [11]. CD155 (Necl5, PVR, Tage4) is an immunoglobulin-like receptor found on certain tumors $[10,12,13]$.
Although functional involvement of TIGIT in altered NK cytotoxicity was not examined, our findings could theoretically be explained as follows. HepG2 and K562 cells express CD112 and CD155 [13] and secrete exosomes [14]. If CD112 or CD155 are enriched on exosomes or secreted as free soluble forms and bind TIGIT, thereby activating this inhibitory receptor, its engagement could depress NK cell cytotoxicity. However, this hypothesis does not explain the observed increase in the percentage of TIGIT $^{+}$NK cells.

Recently it has been shown that K562 cells can secrete exosomes rich in microRNAs, many of which are involved in modulation of Wnt signaling pathways [14], which could change transcription of the gene encoding TIGIT. The role of Wnt signaling has been investigated previously in differentiation of NK cell lineages [15]. It is of interest to study other facets of this pathway in NK cell biology.

In conclusion, our study demonstrates an increase of NK cells expressing inhibitory receptor TIGIT that may decrease the natural cytotoxicity of these cells. It is of interest to determine whether this phenomenon is recapitulated in cancer patients and could represent a novel parameter in assessment of NK cell function.

\section{References}

1 Farag S.S., Caligiuri M.A. Human natural killer cell development and biology // Blood Rev. 2006. - №20. - P. 123-137.

2 Lanier L.L. NK cell receptors // Annu. Rev. Immunol. - 1998. - №16. - P. 359-93. 
3 Vitale M., Cantoni C., Pietra G., Mingari M.C., Moretta L. Effect of tumor cells and tumor microenvironment on NK-cell function // Eur. J. Immunol. - 2014. - №44. - V.6. - P.1582-92.

4 Taketomi A., Shimada M., Shirabe K., Kajiyama K., Gion T., Sugimachi K.Natural killer cell activity in patients with hepatocellular carcinoma A new prognostic indicator after hepatectomy // Cancer. - 1998. - V. 83. - Issue 1. - P. 58-63.

5 Mamessier E., Pradel L.C., Thibult M.-L., Drevet C., Zouine A., Jacquemier J., Houvenaeghel G., Bertucci F., Birnbaum D., Olive D. Peripheral blood NK cells from breast cancer patients are tumor-induced composite subsets // J. Immunol. 2013. - №190. - V.5. - P. 2424-2436.

6 Jin S., Deng Y., Hao J.-W., Li Y., Liu B., Yu Y., Shi F.D., Zhou Q.H. NK cell phenotypic modulation in lung cancer environment // PLoS ONE. - 2014. - №9. - V.10. - e109976.

7 Alter G., Malenfant J.M., Altfeld M. $\mathrm{CD} 107 \mathrm{a}$ as a functional marker for the identification of natural killer cell activity // J. Immunol. Methods. - 2004. - №294. - V.1-2. - P.15-22.

8 Stanietsky N., Mandelboim O. Paired NK cell receptors controlling NK cytotoxicity // FEBS Letters. - 2010. - №584. - P. 4895-4900.

9 Pauken K.E., Wherry E.J. TIGIT and CD226: tipping the balance between costimulatory and coinhibitory molecules to augment the cancer immunotherapy toolkit // Cancer Cell. - 2014. №26. - P. 785-787.
10 Ogita H., Takai Y. Nectins and nectin-like molecules: roles in cell adhesion, polarization, movement, and proliferation // Life. - 2006. - №58. - V.5-6. - P. 334 - 343.

11 Nagumo Y., Iguchi-Manaka A., YamashitaKanemaru Y., Abe F., Bernhardt G., Shibuya A., Shibuya K. Increased CD112 expression in methylcholanthrene-induced tumors in CD155-deficient mice // PLoS. - 2014. - V. 9. - Issue 11. e112415.

12 Atsumi S., Matsumine A., Toyoda H., Niimi R., Iino T., Sudo A. Prognostic significance of CD155 mRNA expression in soft tissue sarcomas // Oncol. Lett. - 2013. - № 5. - V.6. - P.1771-1776.

13 Carlsten M., Norell H., Bryceson Y.T., Poschke I., Schedvins K., Ljunggren H.-G., Kiessling R., and Malmberg K.-J. Primary human tumor cells expressing CD155 impair tumor targeting by down-regulating DNAM-1 on NK cells // J. Immunol. - 2009. - №183. - P. 4921-4930.

14 Feng D.Q., Huang B., Li J., Liu J., Chen X.M., Xu Y.M., Chen X., Zhang H.B., Hu L.H., Wang X.Z. Selective miRNA expression profile in chronic myeloid leukemia K562 cell-derived exosomes // Asian Pac. J. Cancer. Prev. - 2013. №14.- V.12. - p.7501-7508.

15 Aoyama K., Delaney C., Varnum-Finney B., Kohn A.D., Moon R.T., Bernstein I.D. The interaction of the Wnt and Notch pathways modulates natural killer versus $\mathrm{T}$ cell differentiation // Stem Cells. - 2007. - №25 . - V.10. - P. 2488-2497. 